\title{
THE STRUCTURE AND FUNCTION OF THE THYLAKOID MEMBRANE
}

by

\author{
DAVID J. SIMPSON and DITER VON WETTSTEIN
}

Department of Physiology, Carlsberg Laboratory, Gamle Carlsberg Vej 10, DK-2500 Copenhagen Valby

\begin{abstract}
Keywords: Chlorophyll-proteins, electron microscopy, freeze-fracture, gel electrophoresis, grana membranes, photosystem I/II, stroma membranes
\end{abstract}

Thylakoid membranes are laterally differentiated into appressed and non-appressed regions called grana and stroma lamellae. Pure stroma lamellae isolated from wild type maize and barley leaves contain photosystem I and its light-harvesting antennae, the cytochrome $b 6 / f$ complex and coupling factor. Maize stroma lamellae contained only $2 \%$ of the total photosystem II polypeptides found in whole thylakoids, and most of the small amount of the photosystem II light-harvesting complex (LHCII) was associated with photosystem I. These results were consistent with the low rates of photosystem II electron transport and low levels of the high potential form of cytochrome $b$-559. Immune blot assays indicated that about half of the low potential form of cytochrome $b-559$ in stroma lamellae was antigenically distinct from that derived from the high potential form. The amount of LHCII in stroma lamellae could be increased by exposing leaves to bright white light (state 2) prior to the isolation of stroma lamellae. This LHCII caused a $15 \%$ increase in photosystem I antenna size and was different from the LHCII found in grana lamellae, since it lacked a $26 \mathrm{kD}$ polypeptide possibly involved in thylakoid appression. These results demonstrate that the migration of LHCII from appressed to non-appressed lamellae as a result of changes in the relative amounts of energy absorbed by the 2 different photosystems, also occurs in vivo.

The reaction centre core of photosystem I was isolated from barley thylakoids and its molecular weight determined to be $650 \mathrm{kD}$. Attack by various proteases cleaved it into fragments of less than $5 \mathrm{kD}$, although the complex was still photoactive. However, the kinetics of photo-oxidation of P700 under light-limiting conditions were slower after proteolysis, indicating less efficient energy transfer. In a barley mutant lacking photosystem I, a chlorophyll $a$ species absorbing at $689 \mathrm{~nm}$ was lacking, accounting for about 30 molecules out of every 500 in wild type thylakoids.

Finally, a mutant completely lacking photosystem II activity, viridis- $1 / 5$, has been examined. It contained only $4 \%$ of the photosystem II-containing EFs particles found in wild type thylakoids, and lacked a chlorophyll $a$ species absorbing at $683 \mathrm{~nm}$. Immune electron microscopy revealed that the $\alpha$-subunit of cytochrome $b-559$ and the 33 $\mathrm{kD}$ polypeptide of the oxygen evolving complex were correctly located in appressed thylakoids, in spite of the lack of the major photosystem II core polypeptides. A double mutant, lacking both photosystem II and LHCII was found to contain grana, even though its thylakoids lacked the 2 complexes normally associated with maintenance of membrane appression in vivo.

\section{INTRODUCTION}

The photosynthetic membranes or thylakoids of higher plants contain 6 major polypeptide complexes - the photosystem I reaction centre (PSI), the photosystem II reaction centre (PSII), the light-harvesting complex of PSI (LHCI), the

Abbreviations: $\mathrm{CF}_{1 / \mathrm{o}}=$ extrinsic/intrinsic chloroplast coupling factor, $\mathrm{CP}=$ chlorophyll-protein; $\mathrm{DCMU}=$ 3-(3,4-dichlorophenyl)-1,1'-dimethyl urea; $\mathrm{EF}=$ endoplasmic fracture face; $\mathrm{kD}=$ kilodaltons; $\mathrm{LHCI} / \mathrm{II}=$ light-harvesting complex of PSI/II: PF = protoplasmic fracture face; PSI/II = photosystem I/II; s = stacked; $u=$ unstacked. 


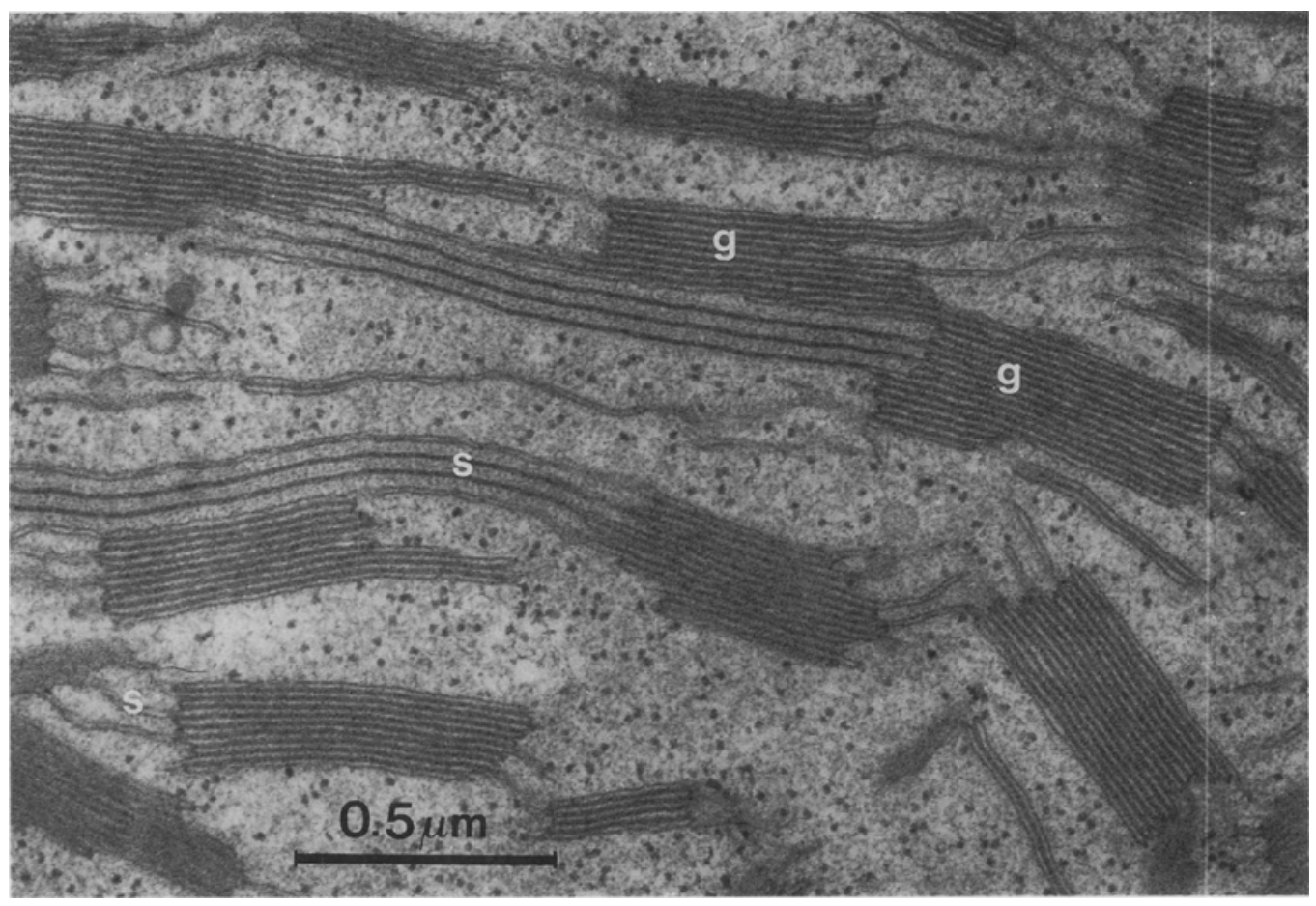

Figure 1. Thin section electron microscopy of a chloroplast showing the internal membranes of thylakoids, which are laterally differentiated into appressed (grana) and non-appressed (stroma) lamellae. In this chloroplast, which is from the PSI-deficient mutant viridis- $z b^{63}$, some of the stroma lamellae have collapsed (13).

light-harvesting complex PSII (LHCII), the cytochrome $b 6 / f$ complex and the chloroplast coupling factor $\left(\mathrm{CF}_{0}\right.$ and $\left.\mathrm{CF}_{1}\right)$. Each of these functional complexes is stable in the absence of one or more of the others, as shown from studies with mutants. Thus the PSII reaction centre is assembled in the absence of LHCII, as in the barley chlorophyll $b$-less mutant chlorina- 2 (12), and LHCII accumulates in the absence of the PSII reaction centre, as in the mutant viridis- $^{1 / 5}$ (17). Although the PSII reaction centre is stable in the absence of the oxygen evolving complex, as shown in manganese-deficient spinach (16) and the Chlamydomonas mutant BF25 (8), the oxygen evolving complex fails to assemble in the absence of PSII.

Over the last 10 years it has become increasingly apparent that the lateral distribution of these different complexes is extremely heterogenous (1). The lateral differentiation of thylakoids into appressed and non-appressed re- gions gives rise to grana and stroma lamellae (Fig. 1). Not only do these membranes have a different structural appearance, as shown by freeze-fracture electron microscopy, but fractionation methods involving detergents or mechanical means, have shown that appressed and non-appressed thylakoids have very different polypeptide compositions. Thus, all of the PSI, LHCI and $\mathrm{CF}_{1}$ is found in the non-appressed thylakoids, while most of the PSII and LHCII is found in appressed thylakoids. The cytochrome $b 6 / f$ complex is evenly distributed between the two types of membranes (4). These results have been independently confirmed by immunoelectron microscopy, using mono-specific antibodies $(20,21)$.

\section{THE STRUCTURE OF THYLAKOIDS}

The structure of the thylakoid membrane is not static and it has long been known that 


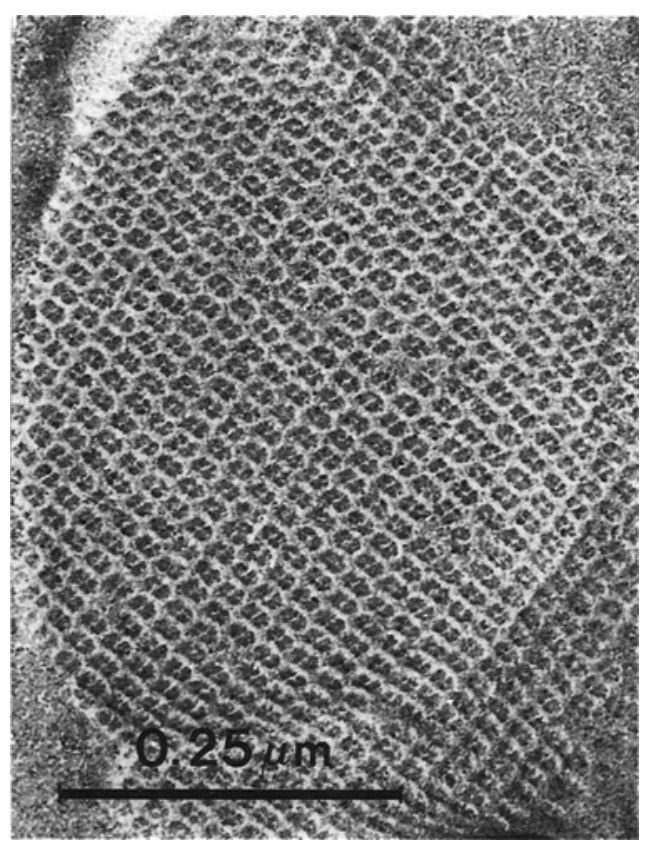

Figure 2. Freeze-etch electron micrograph of the lumenal surface of wild type thylakoids showing regular arrays of the ESs tetrameric particles which are characteristic of this surface, and which contain the extrinsic polypeptides of the oxygen evolving complex (15).

destacking and subsequent randomisation of the distribution of the complexes can be induced by manipulating the ionic environment of isolated thylakoids. Extremely regular particle arrays can be found in situ in appressed thylakoids (Fig. 2), or induced in vitro in destacked thylakoids (9). Of more physiological significance, is the disconnection of LHCII from the PSII reaction centre and its subsequent migration from appressed to non-appressed thylakoid membranes, as a result of its phosphorylation by a membrane-bound kinase (18). This kinase is activated by the redox potential of plastoquinone via the cytochrome $b 6 / f$ complex. The movement of LHCII balances the energy distribution between the two photosystems, ensuring a maximal electron transport rate and photosynthetic efficiency when the plant is exposed to changes in light quality or intensity. This may also provide a mechanism to protect the PSII reaction centre from excessive light, although even under favourable conditions, plants pay a price for oxygenic photosynthesis in the high turnover rate of the PSII reaction centre polypeptide DI. The turnover rate is proportional to the light intensity, and is zero in the dark. Heat stress is also known to cause dynamic changes in the structure of the thylakoid membrane, resulting in the migration of the PSII reaction centre from appressed to non-appressed regions (5).

The complete amino acid sequence of many thylakoid polypeptides has been determined over the last decade, either by direct sequencing of the purified protein, or by deduction from the nucleic acid sequence of the gene, or a combination of these methods. In the case of nuclear encoded polypeptides, cDNA libraries have been screened with antibodies in order to select the desired cDNA clone (19). The complete nucleic acid sequence of chloroplast DNA is now known for tobacco (11), Marchantia (10) and rice (7), and this has led to the identification of previously unknown genes by searching through computer databases for homology with known proteins.

The diagram below (Fig. 3) is a schematic model of the thylakoid membrane and gives the gene name and location of those polypeptides whose gene has been identified and sequenced. In some cases, the gene product has not yet been isolated from thylakoids. There are 9 known polypeptides of the chloroplast coupling factor $\left(\mathrm{CF}_{\mathrm{o}}\right.$ and $\left.\mathrm{CF}_{1}\right)$, or ATPase, and the genes coding for them are designated atp A-I. To date, 8 genes coding for PSI polypetides have been identified and are called $p s a \mathrm{~A}-\mathrm{H}$, and 12 for PSII polypeptides, similarly designated $p s b \mathrm{~A}-\mathrm{L}$. The oxygen evolving complex (OEC) consists of extrinsic and intrinsic polypeptides, and we have given their genes the provisional designation oec $\mathrm{A}-\mathrm{H}$, since the function of all these polypetides has yet to be firmly established. The polypeptides of the cytochrome $b 6 / f$ complex, which are also considered to include plastocyanin (PC), ferredoxin (Fd), ferredoxin nucleotide reductase (FNR) and an FNR-binding subunit, participate in photosynthetic electron transport between PSII and PSI and are encoded by the genes pet A-I. Genes coding for the chlorophyll $a / b$-binding proteins $(c a b)$ have been mapped to 7 different 
a

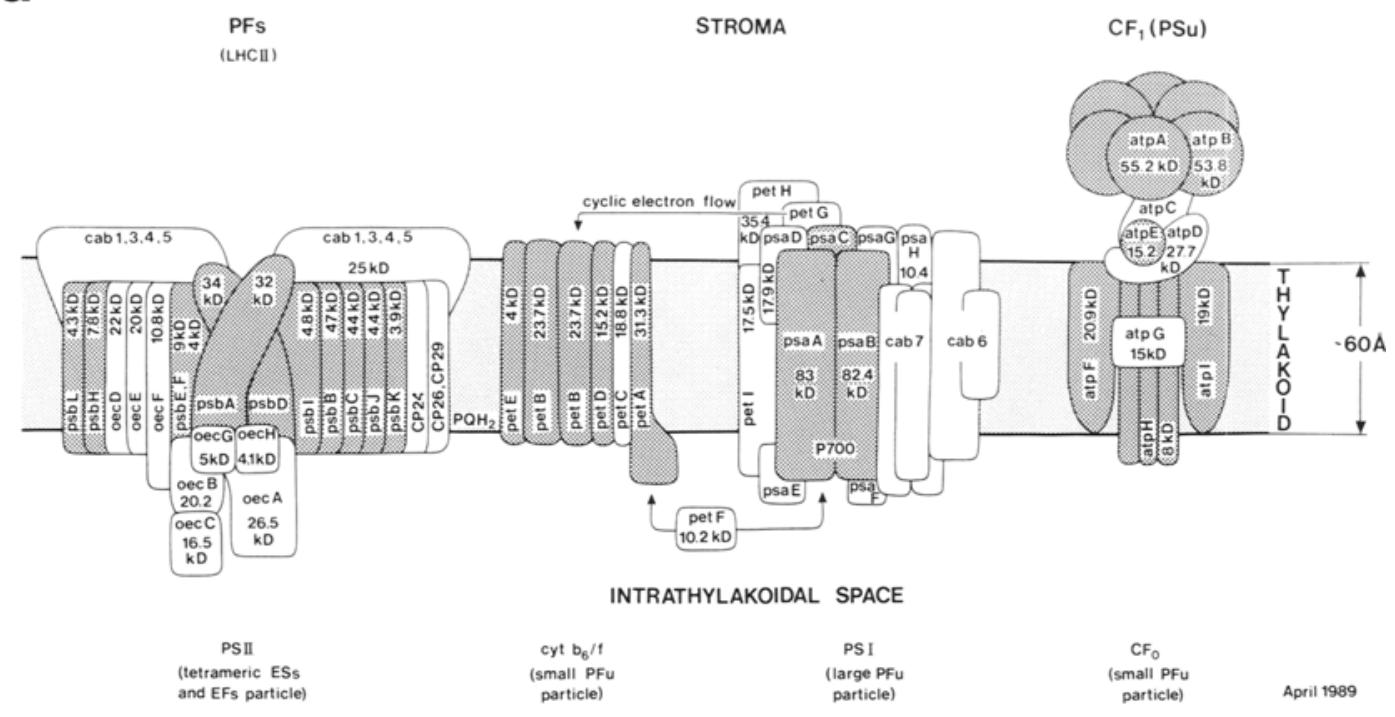

b

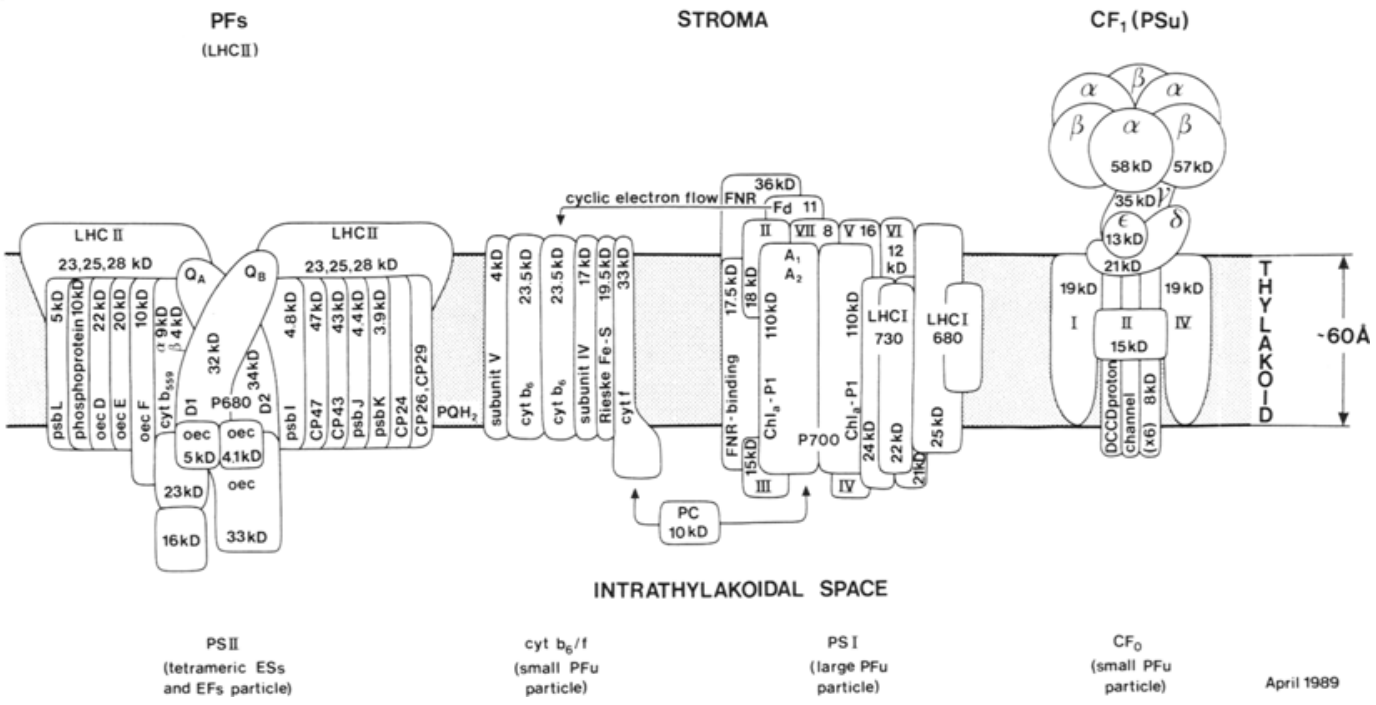

Figure 3. (a) Model of the thylakoid membrane showing the known polypeptide components of the major complexes. The gene name and actual molecular weight are given, where known, and the site of coding is indicated by shading (chloroplast) or clear (nucleus).

(b) Model of the thylakoid membrane showing the name of the individual polypeptide components and their apparent molecular weights determined by denaturing polyacrylamide gel electrophoresis. 


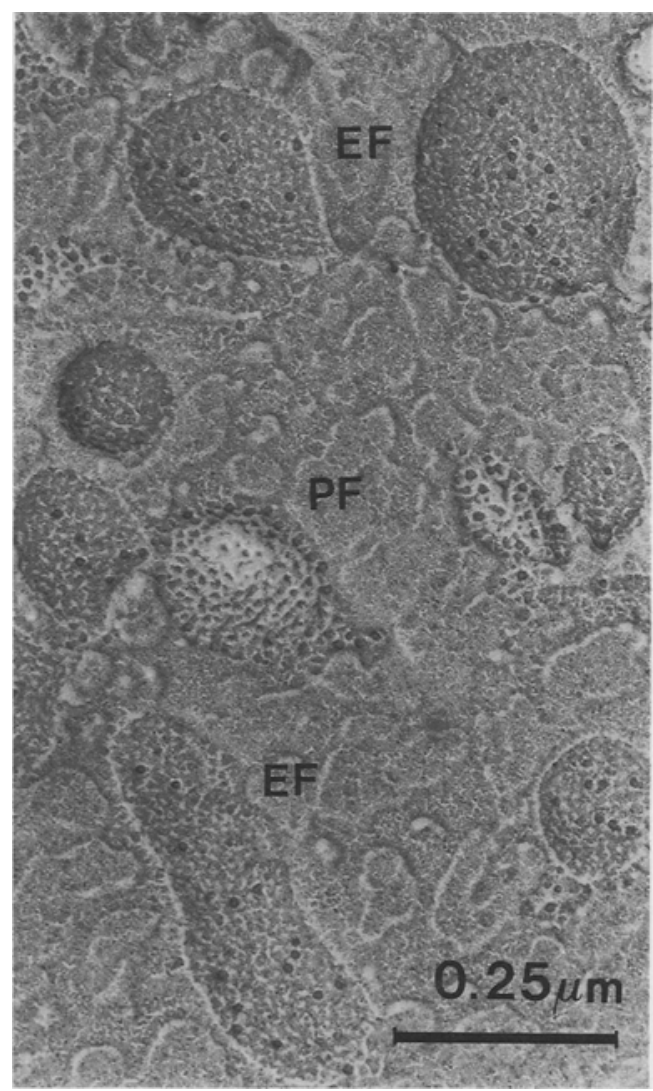

Figure 4. Freeze-fracture electron micrograph of stroma vesicles isolated from dark-adapted maize seedling leaves. Their appearance is identical to that of the EFu and $\mathrm{PFu}$ fracture faces of the unstacked regions of intact thylakoids.

loci on 7 different chromosomes in tomato, and code for two different classes of both LHCI ( $c a b 6$ and 7) and LHCII ( $c a b 1,2,3$ and $c a b 4,5$ ).

While the polypeptides of the ancillary complexes LHCI, LHCII and the oxygen evolving complex all seem to be encoded by nuclear genes, those of the 4 major functional complexes (PSI, PSII, $\mathrm{CF}_{0} / \mathrm{CF}_{1}$ and cytochrome $b 6 / f$ ) are encoded by genes located on chloroplast and nuclear DNA. If one of these polypeptides fails to accumulate in the thylakoid, the other polypeptide components of the functional complex are also usually absent, either due to its failure to assemble, or to an accelerated turnover of the components of a deficient complex. In

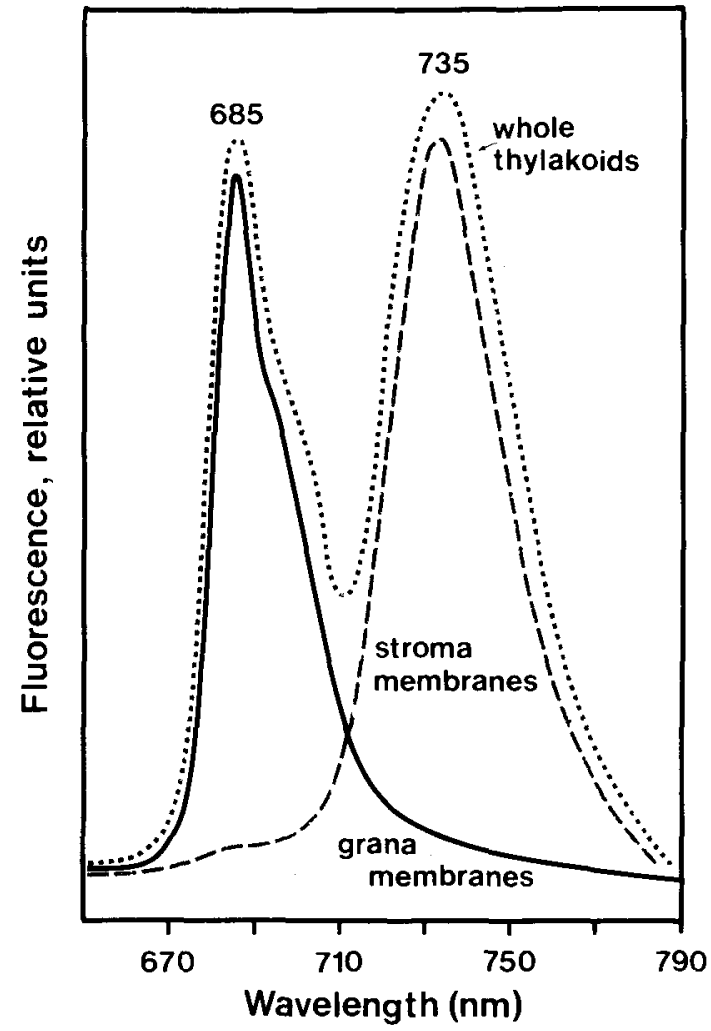

Figure 5. Low temperature $(77 \mathrm{~K})$ fluorescence emission spectra of isolated stroma and grana membranes compared with that of whole thylakoids. The $735 \mathrm{~nm}$ peak comes from LHCI attached to PSI and the 685 and $695 \mathrm{~nm}$ peaks come from LHCII attached to PSII.

addition to the structural genes encoding the individual polypeptides, there are many nuclear genes coding for proteins involved in the transport of nuclear encoded polypeptides into the chloroplast, or required for the processing of the primary transcripts from chloroplast DNA before they can be translated. Mutations in such nuclear genes can exhibit pleiotropic effects on chloroplast composition, or specifically affect the translation of a single chloroplast encoded gene.

\section{STROMA LAMELLAE}

Chloroplast thylakoids can be mechanically fractionated by French press treatment, fol- 
Table I. Composition of barley thylakoids

\begin{tabular}{llll}
\hline Component & stroma lamellae & $\begin{array}{l}\text { wild type } \\
\text { thylakoids }\end{array}$ & $\begin{array}{l}\text { viridis-1/s } \\
\text { thylakoids }\end{array}$ \\
\hline cytochrome $f$ & 1.6 & 1.6 & 1.9 \\
cytochrome $b 6$ & 3.5 & 3.3 & 4.8 \\
cylochrome $b$-559 LP & 2.0 & 3.2 & 3.1 \\
cyochrome $b$-559 HP & 0.004 & 2.4 & 0.12 \\
P700 & 5.2 & 2.2 & 1.9 \\
Chlorophyll $a / b$ & 7.4 & 3.5 & 2.4 \\
\hline
\end{tabular}

*values are in nmoles/mg $\mathrm{Chl}$ (except for $\mathrm{Chl} a / b$ ratio)

lowed by differential centrifugation. This method takes advantage of the greater sensitivity of the non-appressed stroma lamellae to physical disruption, which produces small vesicles that can be separated from the larger vesicles derived from appressed thylakoid membranes (3). The yield is very low, in order to avoid contamination of stroma vesicles by grana vesicles, but the freeze-fracture appearance of isolated stroma vesicles is typical of the unstacked regions of intact thylakoids (Fig. 4). The properties of isolated stroma lamellae differ greatly from those of isolated grana lamellae or whole thylakoids. The $77 \mathrm{~K}$ fluorescence emission spectrum shows a single peak at $735 \mathrm{~nm}$ due to PSI and LHCI, and almost no emission by PSII and LHCII at 685 and $695 \mathrm{~nm}$ (Fig. 5).

The polypeptide composition of stroma lamellae isolated from dark-adapted plants is also very different from that of whole thylakoids (Fig. 6), being dominated by components of $\mathrm{CF}_{1}, \mathrm{PSI}$ and LHCI. Other components of isolated stroma lamellae were measured spectrophotometrically, and are shown in Table I. In comparison with whole thylakoids, stroma lamellae are enriched in P700, the PSI reaction centre, but are depleted in chlorophyll $b$ and severely depleted in the high potential form of cytochrome $b-559(20)$, which is associated with physiologically active PSII reaction centres. The concentration of cytochrome $b 6 / f$ is the same in stroma lamellae as in whole thylakoids, as expected from its even distribution between appressed and non-appressed thylakoids. The amount of the low potential form of cytochrome $b-559$ ( $20 \%$ of whole thylakoids) was twice as much as the amount of the $9.2 \mathrm{kD}$ a-subunit of cytochrome $b-559$ determined by immune blot analysis. This indicates that half of the low potential cytochrome $b-559$ in stroma lamellae is antigenically distinct from that associated with PSII in the high potential form.

Differences in polypeptide composition between stroma lamellae, grana lamellae, and whole thylakoids are reflected in their chlorophyll-protein composition (Fig. 7). Stroma lamellae contain mainly the reaction centre of PSI (CPI) and its light-harvesting complex (LHCI-680 and LHCI-730). In contrast to grana membranes, where all the LHCll can be maintained in the oligomeric form (LCHII ${ }^{* *}$ ) in the presence of $40 \%$ glycerol, the LHCII of stroma lamellae is mostly monomeric after electrophoresis (Fig. 7), but can be stabilised by the addition of $5 \mathrm{mM} \mathrm{MgCl}$ to the sample (2). This indicates that stroma LHCII differs from grana LHCII, and this was confirmed by denaturing gel electrophoresis. LHCII from grana lamellae contains 4 polypeptide components in the 28-30 $\mathrm{kD}$ region and a $26 \mathrm{kD}$ polypeptide which is not found in stroma $\operatorname{LHCII}(2,3)$.

LHCll in stroma lamellae could be connected to PSII or to PSI. We therefore quantified the levels of PSII polypeptides in isolated stroma lamellae by immune blot assay. Whereas $20 \%$ of the total PSII was found in spinach stroma lamellae, only $8 \%$ was found in barley (20), and less than $2 \%$ in maize stroma lamellae (2). These results are shown visually in Figure 8 , where stroma and grana vesicles have been reacted with a monospecific antibody against the PSII component CP47 and labelled with colloidal gold. The intense labelling of the large gramaderived vesicles contrasts with the low level of 


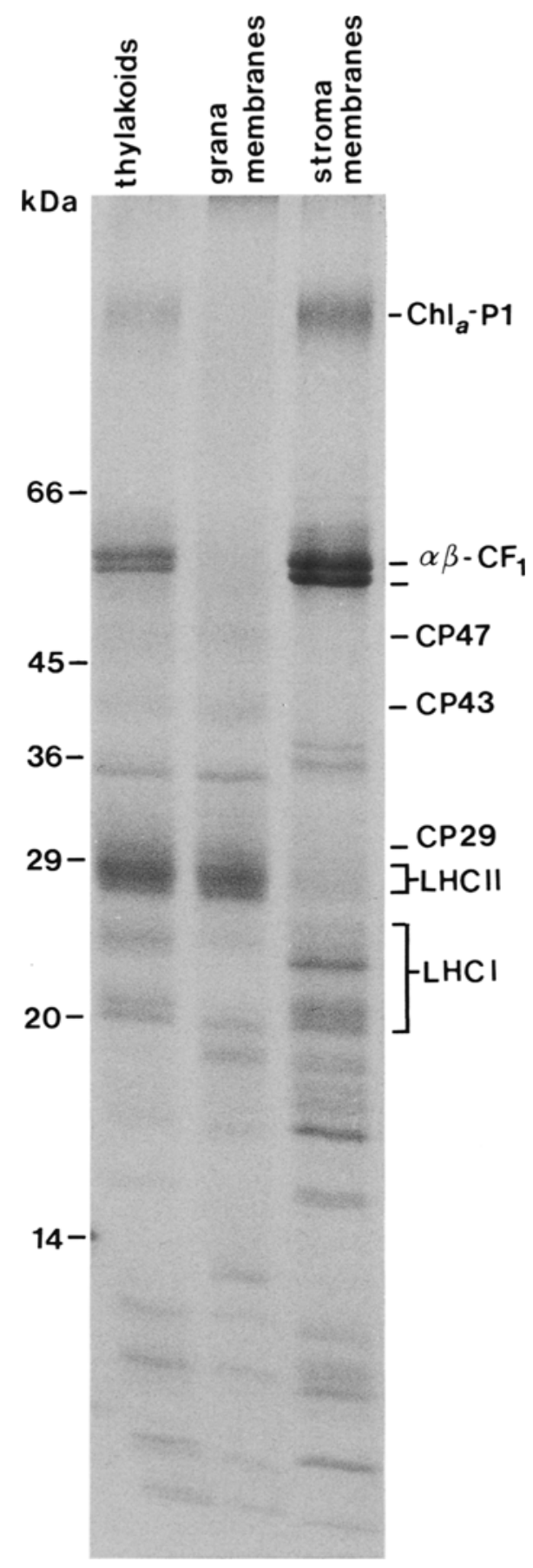

Figure 6. Polypeptide composition of isolated stroma and grana membranes compared with whole thylakoids. The major polypeptides of stroma lamellae are those belonging to PSI, LHCI and the coupling factor complexes, while those of grana membranes are components of the PSII reaction centre and of LHCII. labelling of the small stroma-derived vesicles. The low levels of PSII polypeptides in stroma lamellae were also consistent with a low PSII electron transport rate, which was unusual in being highly resistant to the inhibitor DCMU. These results imply that most of the stroma LHCII must be associated with PSI, and not PSII, and this is consistent with the absence of 685 and $695 \mathrm{~nm}$ fluorescence at $77 \mathrm{~K}$ (Fig. 5). Consequently, there are too few PSII reaction centres in stroma lamellae, especially in maize, for them to constitute the $\mathrm{EFu}$ freeze-fracture particles.

The amount of LHCII in the stroma can be manipulated in vitro by exposing isolated thylakoids to bright white light (state 2) or by dark adaptation (state 1). This is the result of the migration of some of the LHCII from appressed to non-appressed regions. We were interested to see if this phenomenon could also be seen in vivo by adapting whole plants to state 1 or state 2 conditions, and isolating LHCII from stroma lamellae. The stroma LHCII consisted of 4 polypeptides in the $28-30 \mathrm{kD}$ region, all of which could be phosphorylated in vitro. A $26 \mathrm{kD}$ polypeptide, which is a component only of grana LHCII, was not phosphorylated and remained in the appressed lamellae. Under our experimental conditions, about $12 \%$ of the total LHCII migrated into the stroma lamellae, where it became associated with PSI, whose antenna size increased by about $15 \%$. Since no change in the PFu particle density was found, it is possible that this "mobile" LHCII becomes part of the large PFu particles in which the PSI reaction centres are located. PSI in stroma lamellae was isolated as a complex free of both LHCI and LHCII, with a molecular weight of $650 \mathrm{kD}$. It fluoresced at $720 \mathrm{~nm}$ at $77 \mathrm{~K}$ and contained 90-100 molecules of chlorophyll $a$ per photooxidisable $\mathrm{P700}$, with little or no chlorophyll $b$. It was remarkably resistant to attack by protease treatment, and retained photochemical activity despite being cleaved into proteolytic fragments of less than $5 \mathrm{kD}$. There was a loss of $10-30$ molecules of chlorophyll $a$ per P700, depending on the protease and length of proteolysis, and a shift in fluorescence emission maximum from 720 to $677 \mathrm{~nm}$. This was accompanied by a slower photo-oxidation of P700 under light-lim- 


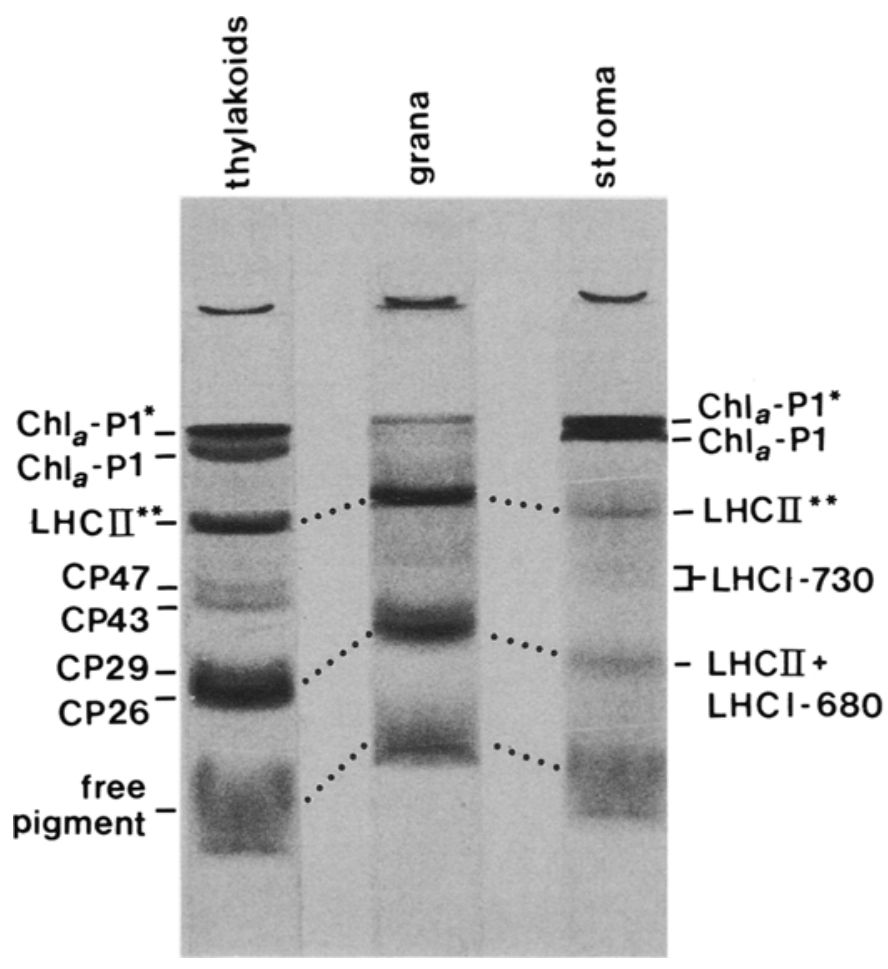

Figure 7. Mild gel electrophoresis of thylakoids, isolated stroma lamellae and grana lamellae. The gels are no stained and the dark bands are visible due to non-covalent association of chlorophyll with specific polypeptides

iting conditions, indicating a disorganisation of the chlorophyll molecules which transfer excitation energy to the reaction centre. Circular dischroism spectra showed a dramatic loss of signal due to carotenoids, indicating that these pigments were accessible to proteases, and therefore located peripherally (6).

The absence of the PSI reaction centre from thylakoids, as in the mutant viridis- $z b^{63}$, resulted in the loss of chlorophyll $a$ absorbing at 689 $\mathrm{nm}$, as shown by fourth derivative spectroscopy and Gaussian deconvolution of the low temperature absorption spectrum. This species accounted for about 30 molecules for every 500 in wild type thylakoids (14).

\section{GRANA LAMELLAE}

Appressed grana lamellae contain mostly PSII and LHCII, as shown by the polypeptide content
(Fig. 6). The major chlorophyll-proteins ar LHCII, CP47, CP43, CP29, CP26 and CP2 (Fig. 7), and result in fluorescence emissio maxima at 685 and $695 \mathrm{~nm}$ (Fig. 5). Th oligomeric form of LHCII in grana is stable $i$ $40 \%$ glycerol in the absence of $\mathrm{MgCl}_{2}$, an prolonged electrophoresis resolves CP26 fror CP29 and CP24, while most of the chlorophyl proteins of stroma lamellae disassociate undt identical conditions (Fig. 9).

The barley mutant viridis- ${ }^{1 / 5}$, which con pletely lacks PSII, has recently been characte: ised (17). It has a high chlorophyll content, an can be distinguished from wild type by its hig yield of red fluorescence under UV light. contained giant grana approximately 5 timt greater in diameter than those of wild type, wit very few intergrana lamellae. The thylakoic lack most of the intrinsic components of th. PSIl core, and contained only $4 \%$ of the normi 
level of EFs particles. The polypeptides of the appressed lamellae therefore consist mostly of LHCII and the cytochrome $b 6 / f$ complex, although normal levels of CP29 and significant amounts of the $33 \mathrm{kD}$ polypeptide of the oxygen evolving complex and the $\alpha$-subunit of the cytochrome $b-559$ were found. The latter 2 polypeptides were shown by immunoelectron microscopy to be located in appressed lamellae, which was unexpected in view of the absence of the core polypeptides of the PSII reaction centre and the EFs particles. These observations are relevant to the way in which the components of the PSII reaction centre move from their site of insertion into the stroma lamellae, to the appressed lamellae.

The cytochrome and $\mathrm{P} 700$ content of viridis ${ }^{1 / 5}$ thylakoids were similar to wild type (Table I) except for the loss of the high potential form of cytochrome $b-559$, which is associated with the loss of PSII. Calculations based on chlorophyll $a / b$ and chlorophyll:P700 ratios indicate that about 60 molecules of chlorophyll $a$ associated with PSII have been lost from viridis- ${ }^{\prime \prime}$. This was confirmed by Gaussian deconvolution of the low temperature absorption spectrum, which showed the loss of a chlorophyll $a$ species absorbing at $683 \mathrm{~nm}$, accounting for 36 out of every 500 molecules in wild type thylakoids (14).

A double mutant was produced between viridis ${ }^{I I S}$ and chlorina- $2^{2800}$ to determine if thylakoid stacking could occur in vivo in the absence of both LHCII and PSII. The recombinant was examined by thin section electron microscopy and found to contain grana with an average of 3.8 discs and a diameter about 4 times that of wild type. The polypeptides of these appressed membranes must consist mostly of the cytochrome $b 6 / f$ complex, and it will be interesting to see if a specific polypeptide (e.g.
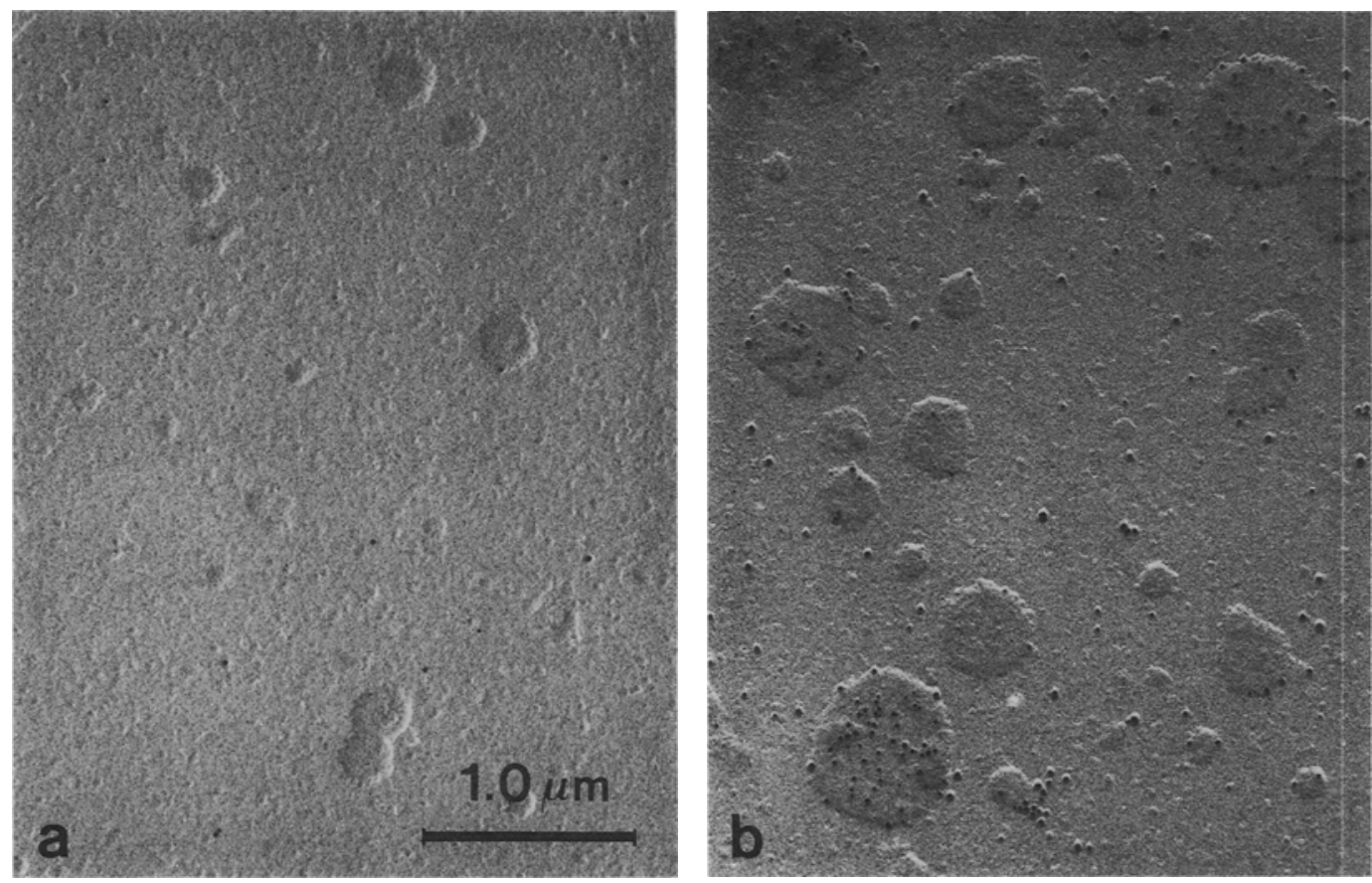

Figure 8. Immunogold labelling of thylakoid vesicles derived from stroma lamellae (a) and stroma plus grana lamellae (b). Vesicles have been labelled "on grid" with an antibody monospecific against the PSII core polypeptide CP43. Binding of the antibody has been visualised by reacting the antibody with protein $A$ bound to colloidal gold. Note the extremely low level of labelling of the small stroma lamellae vesicles in comparison with the heavy labelling of the large grana vesicles (20). 


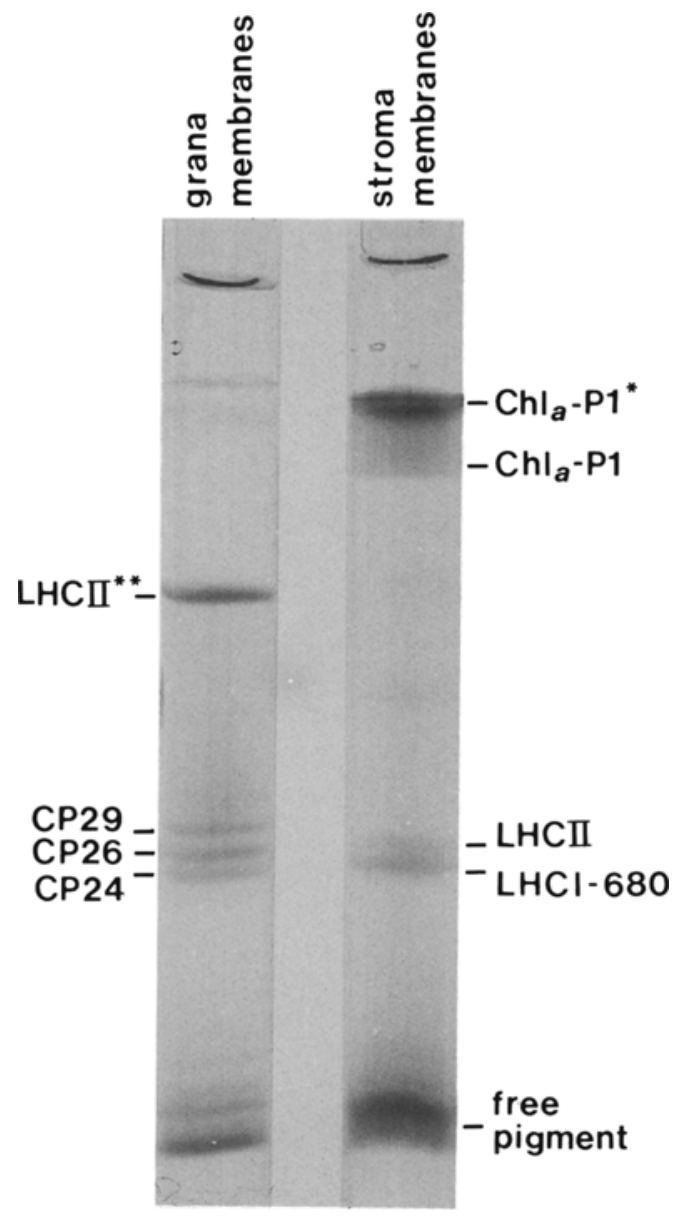

Figure 9. Mild electrophoresis of isolated grana lamellae for an extended time. In the grana membranes, LHCII is maintained as the oligomer, allowing the resolution of CP29, CP26 and CP24. Many of the original chlorophyll-protein bands of the stroma lamellae are faint or absent.

the $26 \mathrm{kD}$ polypeptide of LHCII) mediates membrane appression in these thylakoids.

\section{ACKNOWLEDGEMENTS}

This work was carried out with the collaboration of Drs R. BASSI, U. HINZ and O. VALLON at the Department of Physiology, Carlsberg Laboratory. We are grateful to JEAN SAGE, BENTE JENSEN and KIRSTEN KRISTIANSEN for expert technical assistance, and to NINA RASMUSSEN and ANN-SoFI STEINHOLTZ for drawing and photography.

\section{REFERENCES}

1. ANDERSON, B. \& J.M. ANDERSON: Lateral heterogeneity in the distribution of chlorophyll-protein complexes of the thylakoid membrane of spinach chloroplasts. Biochim. Biophys. Acta 593, 427440 (1980)

2. BASSI, R., G. Giacometti \& D.J. Simpson: Characterization of stroma membranes from Zea mays $L$. chloroplasts. Carlsberg Res. Commun. 52, 221232 (1988a)

3. BASSI, R., G. Giacometti \& D.J. Simpson: Changes in the organization of stroma membranes induced by in vivo state 1 - state 2 transition. Biochim. Biophys. Acta 935, 165-192 (1988b)

4. Cox, R. \& B. ANDERSON: Lateral and transversal organization of cytochromes in the chloroplast thylakoid membrane. Biochem. Biophys. Res. Commun. 103, 1336-1342 (1981)

5. Gounaris, K., A.R.R. Brain, P.J. Quinn \& W.P. WILliams: Structural reorganization of chloroplast thylakoid membranes in response to heat stress. Biochim. Biophys. Acta 766, 198-208 (1984)

6. HiNZ, U.G. \& D.J. SimPSON: The protease-resistant core of the reaction centre protein of photosystem I. Carlsberg Res. Commun. 53, 321-330 (1988)

7. Hiratsuka, J., H. Shimada, R.F. Whittier, T. ISHIBASHI, M. SAKamoto, M. MORI, M. Kondo, Y. HONJI, C.R. SUN, B.Y. MENG, Y.Q. Li, A. KANNO, Y. Nishizawa, A. Hirai, K. Shinozaki \& M. Sugiura: The complete sequence of the rice (Oryza sativa) chloroplast genome. In press (1989)

8. Mayfield, S.P., M. Rahire, G. FranK, H. Zuber \& J.-D. Rochalx: Expression of the nuclear gene encoding oxygen-evolving enhancer protein 2 is required for high levels of photosynthetic oxygen evolution in Chlamydomonas reihardtii. Proc. Natl. Acad. Sci. USA 84, 749-753 (1987)

9. MaChOLd, O., D.J. SimPSON \& G. Høyer-HANSEN: Correlation between the freeze-fracture appearance and polypeptide composition of thylakoid membranes in barley. Carlsberg Res. Commun. 42, 499-516 (1977)

10. Ohyama, K, H. Fuzuzawa, T. Kohchi, H. Shirai, S. Sano, K. Umesono, Y. Shiki, M. Takeuchi, $Z$. Chang, S. Aota, H. Inukuchi \& H. Ozeki: Chloroplast gene organization deduced from complete sequence of liverwort Marchantia polymorpha chloroplast DNA. Nature 322, 572-574 (1986) 
11. Shinozaki, K., M. Ohme, M. Tánaka, T. WaKaSugi, N. Hayashida, T. Matsubayashi, J. ChunWONGSE, J. OBOKaTA, K. YamagUCHI-ShINOZAKI, C. Ohto, K. Torazawa, B.Y. Meng, M. Sugita, H. DENo, T. Kamogashira, K. Yamada, J. Kusuda, F. Takaiwa, A. Kata, N. Tohdoh, H. Shimada \& M. SUGIURA: The complete nucleotide sequence of the tobacco chloroplast genome: its gene organization and expression. EMBO J. 5, 2043-2049(1986)

12. Simpson, D.J.: Freeze-fracture studies on barley plastid membranes III. Location of the light-harvesting chlorophyll-protein. Carlsberg Res. Commun. 44, 305-336 (1979)

13. SIMPSON, D.J.: Freeze-fracture studies on barley plastid membranes VI. Location of the P700 chlorophyll $a$-protein 1. Eur. J. Cell Biol. 31, 305-314 (1983)

14. SimpSON, D.J.: Low temperature absorption spectroscopy of barley mutants. Gaussian deconvolution and fourth derivative analysis. Carlsberg Res. Commun. 53, 343-356 (1988)

15. SIMPSON, D.J. \& D.J. ANDERSON: Extrinsic polypeptides of the chloroplast oxygen evolving complex constitute the tetrameric ESs particles of higher plant thylakoids. Carlsberg Res. Commun. 51, 467-474 (1986)

16. SimpSon, D.J. \& S.P. RoBinSon: Freeze-fracture ultrastructure of thylakoid membranes in chloroplasts from manganese-deficient plants. Plant
Physiol. 74, 735-741 (1984)

17. Simpson, D.J., O. Vallon \& D. Von Wettstein: Freeze-fracture studies of barley plastid membranes VIII. In viridis-115, a mutant completely lacking photosystem II, OEE 1 and the $\alpha$-subunit of cytochrome $b-559$ accumulate in appressed thylakoids. Biochim. Biophys. Acta 975, 164-174 (1989)

18. Staehelin, L.A. \& C.J. ARNTZEN: Regulation of chloroplast membrane function: protein phosphorylation changes the spatial organization of membrane components. J. Cell Biol. 98, 1327-1337 (1983)

19. Tittgen, J., J. Hermans, J. Steppuhn, T. Jansen, C. JANSSON, B. ANDERSON, R. NEChUSHTAI, N. NELSON \& R.G. HERRMANN: Isolation of cDNA clones for fourteen nuclear-encoded thylakoid membrane proteins. Mol. Gen. Genet. 204, 258-265 (1986)

20. Vallon, O., G. HøYeR-HaNSEN \& D.J. SimpSON: Photosytem II and cytochrome $b-559$ in the stroma lamellae of barley chloroplasts. Carlsberg Res. Commun. 52, 405-421 (1987)

21. VAlLON, O., F.A. WollmanN \& J. Olive: Lateral distribution of the main protein complexes of the photosynthetic apparatus in Chlamydomonas reinhardtii and in spinach: an immunocytochemical study using intact thylakoid membranes and a PSII-enriched membrane preparation. Photobiochem. Photobiophys. 12, 203-220 (1986) 